\title{
Thio- und Seleno-Formaldehyde aus Alkalichalkogeniden und Methylenchlorid
}

\author{
Max Schmidt, Kurt Blaettner, Peter Kochendörfer und Heinz Ruf \\ Institut für Anorganische Chemie der Universität Würzburg \\ Herrn Prof. Dr. Kautsky zum 75. Geburtstag gewidmet \\ (Z. Naturforschg. 21 b, 622-625 [1966]; eingegangen am 2. April 1966)
}

\begin{abstract}
Die Umsetzung von Methylenchlorid mit Alkalisulfid führt in alkoholisch-wäßrigem Medium zu Polythioformaldehyden. In Abhängigkeit vom Wassergehalt erhält man bevorzugt hochpolymere oder niedermolekulare Formen. Dabei wurden neben dem bekannten Trithian erstmals ein tetramerer und pentamerer Thioformaldehyd erhalten. Die spontane thermische Polymerisation eines „Rohtetrathians“ wird beschrieben. Die Verwendung von $\mathrm{Na}_{2} \mathrm{Se}$ bzw. $\mathrm{H}_{2} \mathrm{Se}$ anstelle von $\mathrm{Na}_{2} \mathrm{~S}$ liefert verschiedene polymere Methylenselenide.
\end{abstract}

Bereits seit etwa 35 Jahren wird die Umsetzung bifunktioneller Halogenkohlenwasserstoffe (bes. 1.2Äthylenchlorid) mit Alkalisulfiden (bes. $\mathrm{Na}_{2} \mathrm{~S}_{4}$ ) nach

$$
\begin{aligned}
n \mathrm{Cl}-\left(\mathrm{CH}_{2}\right)_{\mathrm{x}}-\mathrm{Cl}+n \mathrm{Na}_{2} \mathrm{~S}_{\mathrm{y}} \rightarrow & \left.\rightarrow\left(\mathrm{CH}_{2}\right)_{\mathrm{x}}-\mathrm{S}_{\mathrm{y}}-\right]_{\mathrm{n}}
\end{aligned}
$$

praktisch zur Darstellung polymerer Kunststoffe herangezogen ${ }^{1}$. Wir haben uns eine systematische Untersuchung solcher Umsetzungen vorgenommen und dafür zunächst die beiden Grundglieder der Ausgangsmaterialien nach Gl. (1) $(x=y=1)$ ausgewählt.

\section{Polymethylensulfide}

Nach

$$
n \mathrm{CH}_{2} \mathrm{Cl}_{2}+n \mathrm{Na}_{2} \mathrm{~S} \rightarrow 2 n \mathrm{NaCl}+\left(\mathrm{CH}_{2} \mathrm{~S}\right)_{n}
$$

ist bei der Reaktion von Methylenchlorid mit Natriumsulfid als "Grundglied der Thiokole" monomer nicht beständiger Thioformaldehyd zu erwarten.

Die trimere Form (1.3.5-Trithiacyclohexan, „Trithian“) wurde bereits 1868 durch säurekatalysierte Umsetzung von Formalin mit Schwefelwasserstoff dargestellt ${ }^{2}$ und ist seither genau untersucht worden ${ }^{3}$, in jüngster Zeit besonders als potentielles Ausgangsmaterial für hochpolymere Methylensulfide. Wahrscheinlich handelte es sich bei dem bereits 1863 aus $\mathrm{CH}_{2} \mathrm{I}_{2}$ mit $\mathrm{Na}_{2} \mathrm{~S}$ erhaltenen „Methylensulfür" ${ }_{4}$ ebenfalls um Trithian. Auch verschiedene hochpolymere (unlösliche) Produkte der Zusammen-

1 M. B. Berenbaum u. J. R. Panek, „The Chemistry and Application of Polysulfide Polymers", Thiokol Chem. Co., Trenton, Sept. 1958 - vollst. Literaturübersicht.

2 A. W. von Hofmann, Liebigs Ann. Chem. 145, 360 [1868].

${ }^{3}$ Literaturübersicht: Dissertation K. Blaettner, Univ. München 1960. setzung $\left(\mathrm{CH}_{2} \mathrm{~S}\right)_{n}$ sind beschrieben. Sie zersetzen sich je nach Darstellungsverfahren zwischen $\sim 160^{\circ}$ bis $220^{\circ}$.

Gl. (2) ließ sich in der Tat experimentell einfach verifizieren, sobald eine homogene Phase vorlag (wäßrige Sulfidlösungen reagieren auch in der Wärme trotz heftigen Rührens nicht mit Methylenchlorid). Dadurch wurde ein bequemes Darstellungsverfahren sowohl für Trithian als auch für hochpolymere Thioformaldehyde erschlossen ${ }^{6}$. Als Homogenisierungsmittel dient Äthanol oder Methanol. Das Reaktionsgemisch wird einige Stdn. erwärmt. Die Isolierung der Produkte ist einfach. Der Wassergehalt des Gemisches bestimmt das Verhältnis von gebildetem niedermolekularem zu hochpolymerem Produkt. Ist kein Wasser vorhanden, entsteht nur $\left(\mathrm{CH}_{2} \mathrm{~S}\right)_{n}$ als weißes, in allen Lösungsmitteln unlösliches Pulver vom Schmp. $220-230^{\circ}$ (unter Zersetzung) (Ausbeute 60-80\%). Bei einem Wassergehalt von $50 \%$ erhält man unter sonst gleichen Bedingungen keine unlöslichen Produkte, sondern hauptsächlich $\left(\mathrm{CH}_{2} \mathrm{~S}\right)_{3}$ in $\sim 75$-proz. Ausbeute. Bei einem $\mathrm{H}_{2} \mathrm{O}$-Gehalt von $10 \%$ ist das Verhältnis Trimer : Polymer $\sim 1: 4$, bei $20 \% \mathrm{H}_{2} \mathrm{O}$ bereits $3: 2$.

Die Umsetzung in wäßrigem Alkohol liefert neben Trithian noch weitere lösliche Verbindungen. Aus dem Gemisch konnte zuerst als bisher unbekannter niedermolekularer Polythioformaldehyd in $~ 15$. proz. Ausbeute 1.3.5.7-Tetrathiacyclooctan, „Tetrathian“", $\left(\mathrm{CH}_{2} \mathrm{~S}\right)_{4}$ isoliert werden ${ }^{6}$. Dieser neue tetramere Thioformaldehyd entspricht einem $\mathrm{S}_{8}$-Ring, in

4 A. Husemann, Liebigs Ann. Chem. 126, 293 [1863].

${ }^{5}$ Literaturübersicht: Dissertation P. Kochendörser, Marburg 1964.

6 Vorläufige Mitteilung: M. Schmidt u. K. BLaettner, Angew. Chem. 71, 407 [1959]. 
dem vier Schwefelatome symmetrisch durch vier $\mathrm{CH}_{2}$ Gruppen ersetzt sind. In reiner Form bildet er farblose, nadelförmige monokline (Raumgruppe $\mathrm{C}_{2}^{5} \mathrm{R}^{\text {- }}$ $\mathrm{P} 2_{1} /$ c) Kristalle vom Schmp. $48,5^{\circ}$, die in organischen Lösungsmitteln sehr gut (in Benzol z. B. $\sim 20$-mal besser als Trithian), in Wasser aber unlöslich sind; $d=1,54$; IR-Spektrum ${ }^{7}$ : 2859 schw, 2890 Schulter, 1396 sst, 1359 st, 1233 sschw, 1215 sst, 1208 sst, 1150 Schulter, 1147 st, 1139 Schulter, 1111 sschw, 1041 schw, 1008 sschw, 874 st, 814 st, 806 st, 750 sst, 733 sst, 710 st, 680 sschw, $\mathrm{cm}^{-1}$; ${ }^{1} \mathrm{H}-\mathrm{NMR}$-Spektrum ${ }^{8}$ : ein scharfes Signal $-185 \mathrm{~Hz}$ $=3,08 \mathrm{ppm}, \quad \tau=6,92$ (für Trithian $-168 \mathrm{~Hz}=$ $2,80 \mathrm{ppm}, \tau=7,20)$.

Die Eignung des so dargestellten Tetrathians als oligomeres Ausgangsmaterial zur Darstellung von Polythioformaldehyd mit Hilfe von Bortrifluorid wurde kürzlich von Russo et al. ${ }^{9}$ ebenso wie die experimentelle Verifizierung der Gl. (2) ${ }^{10}$ zur Darstellung polymerer Produkte untersucht.

Als weitere niedermolekulare Form von Thioformaldehyd haben wir bei der Umsetzung von Methylenchlorid mit alkoholisch-wäßrigen Alkalisulfidlösungen eine pentamere Form, das 1.3.5.7.9. Pentathiacyklodekan $\left(\mathrm{CH}_{2} \mathrm{~S}\right)_{5}$ isolieren können ("Pentathian“) ${ }^{5}$, allerdings nur in $\sim 2$-proz. Ausbeute (die Ausbeute an Pentathian nach unserem Darstellungsverfahren konnte kürzlich unabhängig von uns von Russo et al. ${ }^{11}$ auf $\sim 10 \%$ gesteigert werden). Pentathian kristallisiert in farblosen $\mathrm{Na}$ deln vom Schmp. $123^{\circ}$, die in Wasser unlöslich, in Methanol schlecht und in Benzol, Aceton usw. gut löslich sind. IR-Spektrum ${ }^{7}$ : 2959 schw, 2890 schw, 1383 st, 1366 sst, 1353 Schulter, 1221 Schulter, 1208 sst, 1193 sst, 1150 schw, 1125 st, 1055 sschw, 1041 sschw, 862 sst, 796 sst, 745 sst, 738 Schulter, 730 sst, 725 sst, 712 sst, 685 schw, $671 \mathrm{schw}, \mathrm{cm}^{-1}$; ${ }^{1} \mathrm{H}-N M R-S p e k t r u m$ : ein scharfes Signal $-179,5 \mathrm{~Hz}$ $=2,99 \mathrm{ppm}, \tau=7,01$.

Bei den Versuchen zur Darstellung von Tetrathian und Pentathian fiel immer auch eine allerdings sehr geringe Menge öliger Nebenprodukte an, die kein Chlor, sondern nur C, S und O $(\sim 12 \%)$ enthielten.

7 Perkin-Elmer 137; KBr-Preßling.

8 Varian A $60(60 \mathrm{MHz})$; 5-proz. Lösung in Benzol, TMS extern.

9 M. Russo, L. Mortillaro, C. De Checchi, G. Valle u' ${ }^{*} \cdot$ Maмmi, Polymer Letters 3, 501 [1965].

10 M. Russo, L. Mortillaro, C. De Checchi u. L. Credali, Gazz. Chim. Ital. 95, 448 [1965].
Es handelt sich dabei wohl um polymere Thioäther, in denen der Schwefel teilweise durch Sauerstoffbrücken ersetzt ist. Diese Nebenprodukte machen wir für ein interessantes Phänomen verantwortlich, das hier kurz beschrieben sei: Rohes Tetrathian (Schmelzbereich $39-44^{\circ}$ ) sollte durch Vakuumdestillation gereinigt werden (eine kleine Probe konnte bei $\sim 130^{\circ} / 1 \mathrm{~mm}$ destilliert werden). Bei einem Ansatz von $50 \mathrm{~g}$ erstarrte die wasserklare Schmelze jedoch bei einer Badtemperatur von $\sim 80^{\circ}$ schlagartig zu einer harten, elfenbeinartigen Masse der exakten Zusammensetzung $\left(\mathrm{CH}_{2} \mathrm{~S}\right)_{n}$. Dieser polymere Thioformaldehyd schmilzt bei $245-247^{\circ}$ zu einer klaren Schmelze ohne Zersetzungserscheinungen, die erst oberhalb $265^{\circ}$ zu beobachten sind (Braunfärbung) $d=1,586$. Er ist unlöslich in allen untersuchten Lösungsmitteln mit Ausnahme einer geringfügigen Löslichkeit in siedendem Dimethylsulfoxid, aus dem er beim Abkühlen aber sofort wieder in Flocken ausfällt. Vorläufige Untersuchungen haben ergeben (Weitwinkel Streuaufnahmen bei verschiedenen Temperaturen), daß das Produkt praktisch bis zum Schmelzpunkt hochkristallin ist. Mit reinstem Tetrathian war diese thermische Polymerisation nicht wiederholbar. Pentathian schmilzt bei sehr raschem Aufheizen bei $123^{\circ}$. Wird es langsam erwärmt, dann schmilzt es meist gar nicht, sondern zersetzt sich erst oberhalb $\sim 230^{\circ}$, ist also ebenfalls in höher polymere Formen übergegangen. Wahrscheinlich ist auch dafür ein sehr kleiner Gehalt an den vorhin erwähnten sauerstoffhaltigen Verunreinigungen verantwortlich, weil diese thermische Polymerisation um so seltener auftritt, je öfter der pentamere Thioformaldehyd umkristallisiert wird (allerdings konnten wir sie auch bei völlig analysenreinen Produkten nicht mit Sicherheit ausschließen).

\section{Polymethylenselenide}

Die säurekatalysierte Umsetzung von Formaldehyd mit Selenwasserstoff ${ }^{12,13}$ bzw. die Einwirkung „freier Methylenradikale“ auf einen Selenspiegel ${ }^{14}$

11 M. Russo, L. Martillaro, L. Credali u. C. De Checchi, Polymer Letters 3, 455 [1965].

12 L. Vanino u. A. Schinner, J. prakt. Chem. (2) 91, 121 [1915].

13 H. J. Bridger u. R. W. Pittmann, J. chem. Soc. [London] 1371 [1950].

14 T. G. Pearson, R. H. Purcelt u. G. S. Saigh, J. chem. Soc. [London] 415 [1938]. 
liefert noch wenig charakterisierte Selenoformaldehyde verschiedener Molekülgrößen. Wir haben solche Verbindungen nach Gl. (2) unter Verwendung von $\mathrm{Na}_{2} \mathrm{Se}$ bzw. $\mathrm{H}_{2} \mathrm{Se}$ anstelle von $\mathrm{Na}_{2} \mathrm{~S}$ erhalten. In wäßrig-alkoholischem Medium (Stickstoffatmosphäre wegen der leichten Oxydierbarkeit des Selenids) wurden nur uneinheitliche, meist schmierige Produkte erhalten. In wasserfreiem Äthanol entstand ein Selenformaldehyd $\left(\mathrm{CH}_{2} \mathrm{Se}\right)_{\mathrm{x}}$ (Ausbeute $45 \%)$, der unlöslich ist in Wasser, Alkohol, Äther, Dioxan, Schwefelkohlenstoff und Petroläther. Die geringfügige Löslichkeit in siedendem Benzol ( 1,5 g/l) ermöglicht eine Reinigung durch Umkristallisieren. Die schwachgelben Kristallnadeln (Schmp. $213^{\circ}$ ), die im Gegensatz zu älteren Literaturangaben ${ }^{13}$ nicht lichtempfindlich, sondern monatelang unverändert haltbar sind, verbrennen beim Erhitzen mit fahlblauer Flamme. Wir vermuten, daß unsere Verbindung identisch ist mit dem früher auf anderem Weg ${ }^{13}$ erhaltenen Selenoformaldehyd (Schmp. 210 ${ }^{\circ}$ ), doch konnten wir das dafür angegebene Mol.-Gew. (ebullioskopisch in Xylol) für $\left(\mathrm{CH}_{2} \mathrm{Se}\right)_{3}$ nicht reproduzieren.

Neben dem beschriebenen Selenoformaldehyd fiel bei der Umsetzung von $\mathrm{CH}_{2} \mathrm{Cl}_{2}$ mit $\mathrm{Na}_{2} \mathrm{Se}$ in Äthanol noch ein öliges, in organischen Lösungsmitteln gut lösliches Gemisch von Methylenseleniden an, in denen die Selenbrücken teilweise durch Sauerstoffbrücken ersetzt sein müssen (Selengehalt $\sim 75 \%$, Theorie $84,9 \%$ ) und die den oben beschriebenen, thermisch polymerisierbaren Schwefelverbindungen entsprechen. Ein ähnliches oder gleiches „goldgelbes, stinkendes Öl“ hatten wohl auch Bridger und Pittmann ${ }^{13}$ in Händen, ohne es weiter zu untersuchen. Aus dem Öl lassen sich im Vakuum zwischen $60-90^{\circ}$ geringe Mengen einer gelben Flüssigkeit (Selengehalt $71,3 \%$ ) abdestillieren; die Hauptmenge polymerisierte dabei aber zu einem in allen Lösungsmitteln unlöslichen, blaßgelben Polymethylenselenid.

Läßt man das ölige Gemisch mehrere Tage lang bei Zimmertemperatur im Hochvakuum stehen, dann destilliert wenig widerlich riechende, gelbe Flüssigkeit (nicht weiter untersucht) in die mit flüssiger Luft gekühlte Falle. Aus dem zurückbleibenden Öl $(\mathrm{Se}=78 \%)$ scheiden sich Kristalle $(\mathrm{Se}=82 \%)$ aus. Eine kryoskopische Mol.-Gew.-Bestimmung in Benzol ergibt für das Öl $284\left(\left[\mathrm{CH}_{2} \mathrm{Se}\right]_{3}=279\right)$ und für die Kristalle $467\left(\left[\mathrm{CH}_{2} \mathrm{Se}\right]_{5}=465\right)$.
Die Umsetzung von Methylenchlorid mit Selenwasserstoff bei Gegenwart von Triäthylamin als HCl-Acceptor im Bombenrohr bei Zimmertemperatur liefert im Verlauf von 8 Tagen in 32-proz. Ausbeute gelben, polymeren, in allen untersuchten Lösungsmitteln unlöslichen, allerdings nicht sehr reinen Selenoformaldehyd vom Schmelzbereich (unter Zersetzung) von $157-177^{\circ}$.

\section{Beschreibung der Versuche}

1. Darstellung von polymerem Thioformaldehyd: Eine Lösung von $13,2 \mathrm{~g}(0,23 \mathrm{Mol}) \mathrm{NaHS}$ in $170 \mathrm{ml}$ Äthanol (dargestellt nach l.c. ${ }^{15}$ ) wird mit $7 \mathrm{~cm}^{3}$ $(0,15 \mathrm{Mol}) \quad \mathrm{CH}_{2} \mathrm{Cl}_{2}$ versetzt und das Gemisch unter Rühren $3 \mathrm{Stdn}$. lang auf $50^{\circ}$ erwärmt. Der gebildete weiße Niederschlag wird auf einer Nutsche abgesaugt, mit Wasser ausgekocht und mit verdünnter Salzsäure nachgewaschen. Nach dem Trocknen im Vakuum hinterbleiben $3,4 \mathrm{~g}$ ( $64 \%$ d. Th.) weißes, amorphes Pulver vom Schmp. (unter Zersetzung) $220-230^{\circ},\left(\mathrm{CH}_{2} \mathrm{~S}\right)_{n}$

$$
\text { Ber. C } 26,08 \text { H } 4,35 \text { S 69,57, }
$$$$
\text { Gef. C } 26,1 \text { H } 4,33 \text { S } 69,38 \text {. }
$$

2. Darstellung von Trithian: $120 \mathrm{~g} \mathrm{Na}_{2} \mathrm{~S} \cdot 9 \mathrm{H}_{2} \mathrm{O}$ werden in $120 \mathrm{~cm}^{3}$ Wasser und $200 \mathrm{~cm}^{3}$ Âthanol gelöst und mit $20 \mathrm{ml}$ Methylenchlorid versetzt. Das Gemisch wird vier Stdn. am Rückfluß erhitzt. Der entstandene Niederschlag wird mit Wasser gewaschen und getrocknet: $12 \mathrm{~g}$ Trithian $\left(78,5 \% \mathrm{~d}\right.$. Th.) $\left(\mathrm{CH}_{2} \mathrm{~S}\right)_{3}$

Ber. C 26,08 H 4,35 S 69,57 Mol.-Gew. 138,

Gef. C 25,9 H 4,32 S 69,1 Mol.-Gew. 137.

3. Darstellung von Tetrathian und Pentathian: $240 \mathrm{~g}$ (1 Mol) $\mathrm{Na}_{2} \mathrm{~S} \cdot 9 \mathrm{H}_{2} \mathrm{O}$ werden in $40 \mathrm{~cm}^{3}$ Wasser geschmolzen und dazu $1000 \mathrm{~cm}^{3}$ Methanol und $61 \mathrm{~cm}^{3}$ (1 Mol) Methylenchlorid gegeben. Das Gemisch wird 5 Stdn. lang am Rückfluß erhitzt. Nach dem Absaugen des Niederschlags (Tri-, Penta- und Polythian) wird das Filtrat mit $300 \mathrm{~cm}^{3}$ Wasser versetzt und die Hauptmenge Methanol im Wasserstrahlvakuum abgezogen. Der dabei ausfallende Niederschlag wird abgesaugt, mit Wasser gewaschen und im Vakuum getrocknet. Die Ausbeute des wachsartigen Rohproduktes (Schmelzbereich $\left.39-44^{\circ}\right)$ beträgt $7,6 \mathrm{~g}(16,5 \% \mathrm{~d}$. Th.). Aus $25 \mathrm{~g}$ Rohprodukt (aus mehreren Ansätzen) wurden durch mehrfache fraktionierte Kristallisation aus trokkenem Äther (Einzelheiten s. 1.c. ${ }^{5}$ ) $13 \mathrm{~g}$ reinstes Tetrathian (Schmp. $48,5^{\circ}$ ) und $0,35 \mathrm{~g}$ reinstes Pentathian (Schmp. $\left.123^{\circ}\right)$ isoliert. $\left(\mathrm{CH}_{2} \mathrm{~S}\right)_{4}$ ber. Mol.-Gew. 184, gef. C 26,1, H 4,32, S 69,3, Mol.-Gew. 181. $\left(\mathrm{CH}_{2} \mathrm{~S}\right)_{5}$ ber. Mol.-Gew. 230, gef. C 26,1, H 4,31, S 69,2 , Mol.-Gew. 227. In besserer Ausbeute $(\sim 10 \%)$ läßt sich Pentathian aus dem primär gebildeten Niederschlag durch Extraktion mit heißem Benzol, Eindampfen und Abtrennen vom mit gelösten (in Benzol) Trithian durch Extraktion mit Aceton gewinnen.

15 G. Brauer, Handbuch der präparativen anorganischen Chemie, S. 278. 
4. Thermische Polymerisation des Rohproduktes aus 3: $50 \mathrm{~g}$ Rohprodukt nach 3 wurden in einem an Ölpumpenvakuum angeschlossenen Kolben geschmolzen und (in der Absicht, zu destillieren) weiter erwärmt. Bei einer Badtemperatur von $\sim 80^{\circ}$ erstarrte der Kolbeninhalt schlagartig zu einer sehr harten, elfenbeinartigen Masse, ohne daß dabei ein flüchtiges Produkt abdestillierte. Das harte Polymerisat, das nur durch Zerschlagen des Reaktionskolbens zu isolieren war, hat die Zusammensetzung $\left(\mathrm{CH}_{2} \mathrm{~S}\right)$ : gef. $\mathrm{C} 26,09, \mathrm{H} 4,34$, S 69,51. Ein Mol.-Gew. konnte nicht bestimmt werden. Das Produkt ist unlöslich (auch beim Sieden) in Wasser, Aceton, Äthanol, Äther, Äthylacetat, Benzol, Butanon, Butylacetat, $\gamma$-Butyrolacton, Chlorbenzol, Chloroform, Cyclohexan, Cyclohexanon, Dimethylformamid, Dioxan, Isobutylalkohol, Methanol, Methylenchlorid, Petroläther, Propanol, Schwefelkohlenstoff, Tetrahydrofuran, Tetrachlorkohlenstoff, Toluol und Xylol. In siedendem Dimethylformamid löst es sich etwas, fällt aber beim Abkühlen unter den Siedepunkt wieder aus. Es schmilzt klar bei $245-247^{\circ}$ und zersetzt sich unter Braunfärbung $>265^{\circ}$.

5. Umsetzung von Methylenchlorid mit Natriumselenid: $6 \mathrm{~g} \mathrm{Na} \mathrm{Ne}_{2} \mathrm{Se}$ (dargestellt aus den Elementen in flüssigem Ammoniak ${ }^{16}$ ) wurden in $\mathrm{N}_{2}$-Atmosphäre in $90 \mathrm{~cm}^{3}$ Äthanol gelöst und zusammen mit 4,4 g Methy. lenchlorid unter Rühren $5^{1 / 2}$ Stdn. lang am Rückfluß erhitzt. Der ausgefallene Niederschlag wird abgesaugt, mit heißem Äthanol (die Waschflüssigkeit wird mit dem gelben Filtrat vereinigt) und anschließend mit Wasser gewaschen und getrocknet (Ausbeute $2 \mathrm{~g}=$ $45 \%$ d. Th.). Das in Äther, Athanol, Dioxan, Petroläther und Schwefelkohlenstoff unlösliche Produkt kann aus Benzol (Löslichkeit beim Sieden nur $1,6 \mathrm{~g} / l$ !) in Form hellgelber Nadeln vom Schmp. $213^{\circ}$ erhalten werden.

$\begin{array}{rlll}\left.\left(\mathrm{CH}_{2}\right) \mathrm{Se}\right)_{\mathrm{n}} & & & \\ \text { Ber. } & \text { C } 12,9 & \text { H } 2,2 & \text { Se } 84,9 \text {, } \\ \text { Gef. } & \text { C } 13,33 & \text { H } 2,25 & \text { Se } 84,5 .\end{array}$

16 W. Klemm, H. Sodomann u. P. Langmesser, Z. anorg. allg. Chem. 241, 281 [1939].
Das gelbe alkoholische Filtrat wurde eingedampft, der ölige Rückstand (zur Abtrennung von $\mathrm{NaCl}$ ) in Benzol aufgenommen, die Lösung getrocknet $\left(\mathrm{Na}_{2} \mathrm{SO}_{4}\right)$ und das Lösungsmittel dann abgezogen. Beim Versuch zur Destillation des gelben Öls (im Vakuum) ging ab $\sim 60^{\circ}$ eine geringe Menge gelben Öls über, doch stieg der "Siedepunkt" kontinuierlich an, bis ab $\sim 90^{\circ}$ jede Destillation aufhörte. Die Hauptmenge des Öls war zu einem unlöslichen Polymeren erstarrt (Selengehalt 82,1\%; das überdestillierte Öl 71,3\%). Deshalb wurde nun eine Probe des Öls (nach Abziehen des Benzols) drei Tage lang im Hochvakuum bei Zimmertemperatur belassen. In der mit flüssiger Luft gekühlten Falle fand sich danach eine sehr unangenehm riechende, nicht weiter untersuchte gelbe Flüssigkeit, aus dem zurückbleibenden Öl hatten sich Kristalle abgeschieden. Selengehalt und Molekulargewicht: Öl 78,13\%, 284 (kryoskop. in Benzol), Theorie für $\left(\mathrm{CH}_{2} \mathrm{Se}\right)_{3}=279$; Kristalle 81,82\%, 467 (kryoskop. in Benzol), Theorie für $\left(\mathrm{CH}_{2} \mathrm{Se}\right)_{5}=465$.

6. Umsetzung von Methylenchlorid mit Selenwasserstoff: In einem Bombenrohr wurde auf ein Gemisch von $4 \mathrm{~g}$ Methylenchlorid und $10 \mathrm{~g}$ Triäthylamin überschüssiger Selenwasserstoff kondensiert und das verschlossene Rohr 8 Tage bei Raumtemperatur gelagert. Nach dem Abziehen des überschüssigen Selenwasserstoffs wurde der Rohrinhalt mit Alkohol herausgespült, der Festkörper abgesaugt, mit Wasser gewaschen und getrocknet. Es blieben $1,4 \mathrm{~g}$ gelbes Pulver zurück (32\% d. Th.), das in allen gebräuchlichen Lösungsmitteln unlöslich war und ab $\sim 160^{\circ}$ langsam unter Zersetzung schmolz.

$\left(\mathrm{CH}_{2} \mathrm{Se}\right)_{n}$

$$
\begin{array}{lllll}
\text { Ber. } & \text { C } 12,9 & \text { H } 2,2 & \text { Se } 84,9 \text {, } \\
\text { Gef. } & \text { C } 11,9 & \text { H } 2,16 & \text { Se } 80,46 .
\end{array}
$$

Der Deutschen Forschungsgemeinschaft und dem Fonds der Chemischen Indust rie danken wir herzlich für die finanzielle Unterstützung dieser Untersuchungen. 